Objectives To examine the diagnosis, common workplace allergens and prevention practices in workers seen for patch testing in a tertiary referral centre in Toronto, Canada.

Methods Demographic, clinical, patch test and workplace information were collected for patients seen between 2012 and 2019. Basic descriptive statistics were generated to compare workers in common industries and jobs.

Results Out 3714 patients evaluated, 1261 were diagnosed with occupational skin disease. Comparison across the healthcare, services, manufacturing, automotive and construction sectors revealed differences in diagnosis and causative agents, workplace characteristics and prevention practices. 308 health care workers included 154 nurses, 34 personal support workers, 28 dental workers and 22 cleaners. 90\% had a diagnosis of occupational irritant contact dermatitis and 34\% had occupational allergic contact dermatitis. Dental workers had the highest percentage of allergic contact dermatitis and higher proportions of occupationally relevant rubber (carba mix and thiuram) and methacrylate/acrylate positives on patch testing. They were more likely to work in a small workplace and less likely to take time off work, file a compensation claim or have health and safety training.

Conclusion Collection of detailed work-related descriptors and clinical information in a patch test database facilitates an understanding of the causative agents and the workplace characteristics that may place workers at increased risk for occupational skin disease, providing a focus for prevention activities.

\section{P-393 HAND AND WRIST MUSCULOSKELETAL DISORDERS ASSOCIATED WITH BIOMECHANICAL RISK FACTORS AMONG TUNISIAN WEAVERS}

${ }^{1}$ Asma Kheder, Ben Afia Latifa, Faten Hermassi, Amira Omrane, Taoufik Khalfallah, Lamia Bouzgarrou. 'University of Monastir - School of Medicine, Tunisia

\subsection{6/OEM-2021-EPI.307}

Introduction The informal industry of handmade carpet is particularly developed in the Tunisian central region with use of ancestral tools. The hand and the wrist are continually solicited during the different performed tasks, which may result in musculoskeletal disorders.

Objective This study aimed to assess the biomechanical constraints of the wrist and hand among Tunisian weavers.

Methods A 6 months study was conducted among a representative sample of traditional weavers in a region of central Tunisia. The survey was based on semi-quantitative ergonomic assessment of biomechanical risk factors, with video recordings during a period of full activity (25 to 40 minutes). Each recording was stopped at fixed time intervals and converted into one hundred images. For each image, the postures of the wrists and hands were encoded and analyzed with ERGOROM software. The Gestural Variability Score (GVS) and the subjective BORG scale were used to estimate postural variation and the level of exerted force.

Results The average duration of the working day was $7.45 \pm$ $2.5 \mathrm{~min}$. The weavers spent $57.5 \%$ of the working time with a wrist extension $>30^{\circ}$ with a relatively high Flexion/Extension GVS of $46 \%$. In the coronal plane, radial and ulnar deviation were found during $11.5 \%$ and $9.4 \%$ of the activity time, respectively. Pinching grip of hand tools was spread over $83.4 \%$ of the working time range with a relatively low GVS (22.2\%). According to the BORG scale, $78.5 \%$ of the weavers estimated the force exerted by the hands and wrists as 'medium' to 'strong' during the weaving activity.

Conclusion During artisanal weaving, the hand and the wrist are deviated from the neutral position adopting awkward postures. Moreover, they are exposed to forceful gripping and repetitive movement, which may lead to musculoskeletal injuries. The prevention of these damages should focus on rethinking poorly designed hand tools.

\section{P-394 MUSCULOSKELETAL DISORDERS AMONG MILITARY DENTISTS: SEMI-QUANTITATIVE ERGONOMIC RISK ASSESSMENT}

${ }^{1}$ Kamoun Salma, Olfa Jlassi, Lamia Bouzgarrou, Jilani Gnounou, Adel Amri. ${ }^{1}$ Centre Militaire de Médecine du Travail et de Sécurité Professionnelle, Tunisia

\subsection{6/OEM-2021-EPI.308}

Introduction The activity of the dentist, notably in the military force, is faced like many others the scourge of musculoskeletal disorder (MSD).

Objectives This study aimed to identify the MSD hazards among dentists and identify work situations that can contribute to their appearance and offer appropriate ergonomic remedial measures for their prevention.

Methods This is a semi-quantitative study conducted in a military dental surgery service in Tunis by observing biomechanical constraints during three different dental acts. These observations were then analysed using ERGOROM software.

Results The MSD hazards identified in this study as common to all dentists were strained postures, repetition, static shoulder postures and bad working position. The neck was in more than 40 degrees flexion or extended over $63 \%$ to $95 \%$ of the working time. The shoulders were in sustained contraction over 40 to $72 \%$ of the working time with a low variation suggesting an isometric shoulder contraction. The elbows were in flexion over 56 to $91 \%$ of the working time with low variation flexion/extension. The preferred wrist position was hyper-extension or hyper-flexion over 20 to $64 \%$ of working time and ulnar or radial deviation over 14 to $35 \%$ of working time. Isometric dental instruments grasp spread over almost the whole working time for the three observed dental acts.

Conclusion Our study confirmed biomechanical constraints and MSD hazards to which dentists are exposed and guided us in our preventive approach in order to propose the most adequate and appropriate solutions to the military command.

\section{P-395 WORKPLACE BULLYING AMONG HEALTHCARE WORKERS: PREVALENCE AND IMPACT ON MENTAL HEALTH}

${ }^{1}$ Asma Kheder, Amen Moussa, Naïma Bouattay, Harrathi Chayma, Lotfi Gaha, Taoufik Khalfallah, Ahmed M'halla, Lamia Bouzgarrou. ' University of Monastir - School of Medicine, Tunisia

\subsection{6/OEM-2021-EPI.309}

Introduction Workplace bullying (WPB) is a peculiar form of workplace violence. This behavior affects the healthcare sector in general and particularly intensive care units such as anaesthesiology. 